\title{
SAINT-VENANT TORSION OF A FUNCTIONALLY GRADED CIRCULAR BAR WITH A RADIAL SLIT
}

\author{
A. BAKSA* \\ Institute of Applied Mechanics, University of Miskolc, Hungary \\ [Received: 08 August 2019. Accepted: 21 October 2019] \\ doi: 10.7546/JTAM.50.20.01.08
}

\begin{abstract}
An analytical solution of Saint-Venant's torsion for circular bar with a slit extending in radial direction from the boundary to the axis of bar is presented. The material of the bar is non-homogeneous, isotropic linearly elastic. The shear modulus of the bar is a smooth function of the radial coordinate. This type of the material inhomogeneity is called functionally graded material. The shear modulus as a function of radial coordinate is assumed to be described by a power law function. For arbitrary value of power law index the solution in closed form is obtained for the Prandtl's stress function, shearing stresses, torsion function and torsional rigidity. Results of the presented analytical method are compared with FEM solution.
\end{abstract}

KEY WORDS: Saint-Venant torsion, circular bar, functionally graded material, slit.

\section{INTRODUCTION}

The object of this paper is to obtain the exact solution of the Saint-Venant's torsion for elastic functionally graded bar of circular cross section with radial slit extending from the boundary inward to the axis of bar. The problem of Saint-Venant torsion of homogeneous bar with slits with the aid of membrane analogy is formulated by Nowacki [1-3] and the solutions are deduced from Fedholm integral equations of the first kind. He gave the expressions of Prandtl's stress functions [1,2] and derived the exact expression the torsional rigidity of homogeneous circular bar with one slit [3]. The torsion of homogeneous circular bar with one radial slit is also studied in book written by Arutynyan and Abramyan [4]. Albas developed an exact and an approximate method for the evaluation of the torsional rigidity of homogeneous cross section with slit [5]. Book by Arutynyan and Abramyan [4] does not give the expression of torsion function. Saint-Venant studied the problem of torsion of a homogeneous sector of circle. He gave the expression of the torsion function for circular cross section with a complete radial slit $[6,7]$. Saint-Venant's derivation is based on the solution of a Neumann type boundary value problem concerning with the torsion function.

\footnotetext{
*Corresponding author e-mail: attila.baksa@uni-miskolc.hu
} 


\section{Saint-Venant Torsion of a Functionally Graded Circular Bar with a Radial Slit}

Hematiyan and Estakhrian [15] formulate and approximate-analytical method for the torsion analysis of thin - to moderately thick - walled functionally graded open cross sections with uniform thickness. The shear modulus has variation only thickness direction. The open cross section is decomposed into some straight, circularly curved and end segments. The obtained results are verified by FEM computations [15]. Paper by Arghavan and Hematiyan gives an analytical formulation for torsional analysis of functionally graded hollow tubes. The thickness of all segments of the cross section are the same and the shear modulus change as a power function of the thickness coordinate [13]. Several examples illustrate the accuracy the presented approximate solution [15]. Paper by Horgan and Chan investigates the effects of material inhomogeneity on the torsional response of linearly elastic isotropic bars [12]. The special case of circular bar with shear modulus depending on the radial coordinate only is examined in detailed [12].

In [14], the author deals with the Saint-Venant torsion of non-homogeneous cylindrical bar with arbitrary cross-section. The shear modulus of the non-homogeneous bar is a given function of the Prandt' stress function of the considered cylindrical bar when its material is homogeneous. An integral transformation similar to the Kirchhoff transformation in non-linear heat conduction is used to get the solution of torsional problem [14].

Present paper gives the complete solution of Saint-Venant's torsion of circular bar with a radial slit for functionally graded materials. Functionally graded materials (FGMs) are microscopically inhomogeneous composite materials, in which the volume fraction of two or more materials is varied smoothly and continuously as a function of position along certain dimension(s) of the body from one point to other $[8,9]$. The considered material inhomogeneity is described by a power law function of the radial coordinate. For the arbitrary value of power law index the solution is obtained in closed form for the Prandtl's stress function, shearing stresses, torsion function and torsional rigidity. Results of the presented analytical method are in good agreement with the FEM solution.

\section{Formulation of TORSiOn OF NON-HOMOgEneOUs Circular BaR With} A RADIAL SLIT

The cross section of the considered non-homogeneous cylindrical bar with a longitudinal slit extending in radial direction from the boundary surface to the axis of bar is shown in Fig. 1. The material is assumed to be radially nonhomogeneous and isotropic linearly elastic. The shear modules as a function of radial coordinate is described by a power law function as

$$
G(r)=g\left(\frac{r}{R}\right)^{n},
$$




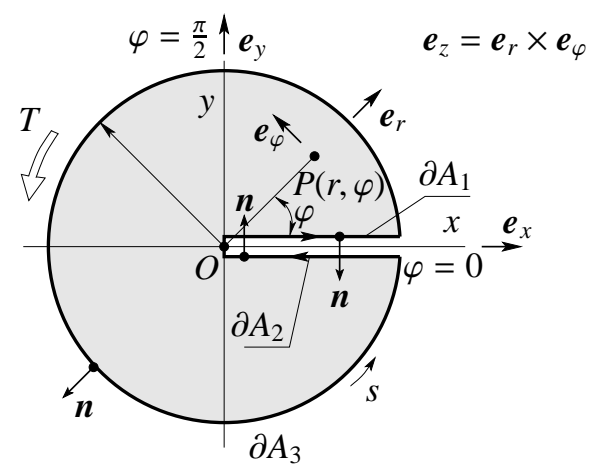

Fig. 1. Radially nonhomogeneous circular cross section with a radial slit.

where $R$ is the radius of the boundary circle of cross section, $g$ is the shear modulus at $r=R$ and $n$ is a material constant (power law index). The cross section of the considered bar is denoted by $A$ and its boundary curve is $\partial A=\partial A_{1} \cup \partial A_{2} \cup A_{3}$, where $\partial A_{3}$ is the curved boundary segment on it $r=R$ and $\partial A_{i}=\{(r, \varphi) \mid 0 \leq r \leq$ $\left.R, \varphi=\varphi_{i}\right\},(i=1,2)$, here $\varphi_{1}=0, \varphi_{2}=2 \pi$ as shown in Fig. 1.

In the case of radially nonhomogeneous bar the Prandtl's formulation of the Saint Venant torsion yields the next boundary value problem $[10,11]$

$$
\frac{\partial}{\partial r}\left(\frac{r}{G(r)}\left(\frac{\partial U}{\partial r}\right)\right)+\frac{\partial}{\partial \varphi}\left(\frac{1}{r G(r)} \frac{\partial U}{\partial \varphi}\right)=-2 r \quad(r, \varphi) \in A
$$

$$
U=0 \quad(r, \varphi) \in \partial A .
$$

Knowing the Prandtl's stress function $U=U(r, \varphi)$ the shearing stresses are obtained as

$$
\tau_{r z}(r, \varphi)=\frac{\vartheta}{r} \frac{\partial U}{\partial \varphi} \quad \tau_{\varphi z}=-\vartheta \frac{\partial U}{\partial r},
$$

where $\vartheta$ is the rate of twist. The applied torque $T$ can be computed from the following equation

$$
T=\vartheta S .
$$

Here, $S$ is the torsional rigidity of the non-homogeneous cross section according to the Saint-Venant's theory of uniform torsion $[4,10,11]$. The value of $S$ is obtained as $[10,11]$

$$
S=2 \int_{A} U \mathrm{~d} A .
$$


86 Saint-Venant Torsion of a Functionally Graded Circular Bar with a Radial Slit

The displacement field in the cylindrical coordinate system $\operatorname{Or} \varphi z$ is as follows (Fig. 1)

$$
\boldsymbol{u}=u \boldsymbol{e}_{r}+v \boldsymbol{e}_{\varphi}+w \boldsymbol{e}_{z},
$$

where $\boldsymbol{e}_{r}, \boldsymbol{e}_{\varphi}$ and $\boldsymbol{e}_{z}$ are the unit vectors of the cylindrical coordinate system used (Fig. 1) and

$$
u=0 \quad v=\vartheta r z \quad w=\vartheta \omega(r \varphi) .
$$

The torsion (warping) function $\omega=\omega(r, \varphi)$ is a solution of the following system of differential equations

$$
\frac{\partial \omega}{\partial r}=\frac{1}{r G(r)} \frac{\partial U}{\partial r} \quad \frac{\partial \omega}{\partial \varphi}=-\frac{r}{G(r)} \frac{\partial U}{\partial r}+r^{2} .
$$

\section{Computations of the Prandtl Stress- and Torsion Functions}

The solution of the boundary-value problem formulated by Eqs. (2) and (3) is looked for as

$$
U_{n}(r, \varphi)=\sum_{k=1}^{\infty} F_{n k}(r) \sin \lambda_{k} \varphi \quad(r, \varphi) \in A \cup \partial A .
$$

The lower index indicates that the shear modulus as a function of radial coordinate is given by Eq. (1), and

$$
\lambda_{k}=\frac{2 k-1}{2} \quad(k=1,2, \ldots) .
$$

It is very easy to show that $U_{n}=U_{n}(r, \varphi)$ satisfies the homogeneous boundary condition on the boundary segment $\partial A_{1} \cup \partial A_{2}$. Substitution of Eq. (10) into Eq. (2) gives

$$
\frac{\mathrm{d}^{2} F_{n k}}{\mathrm{~d} r^{2}}+\frac{1-n}{r} \frac{\mathrm{d} F_{n k}}{\mathrm{~d} r}-\frac{\lambda_{k}^{2}}{r^{2}} F_{n k}=-\alpha_{k} \frac{g}{R^{n}} r^{n},
$$

where

$$
\alpha_{k}=\frac{8}{(2 k-1) \pi} \quad(k=1,2, \ldots) .
$$

To obtain Eq. (12) it was used the next Fourier series representation for $f(\varphi)=2$ $(0<\varphi<2 \pi)$

$$
f(\varphi)=\sum_{k=0}^{\infty} \alpha_{k} \sin \lambda_{k} \varphi .
$$


The solution of differential equation (12) which is bounded at $r=0$ is

$$
F_{n k}(r)=A_{n k} r^{p_{n k}}-\frac{\alpha_{k} g r^{n+2}}{\left(2 n+4-\lambda_{k}^{2}\right) R^{n}},
$$

where

$$
p_{n k}=\frac{n}{2}+\sqrt{\left(\frac{n}{2}\right)^{2}+\lambda_{k}^{2}} \quad(k=1,2, \ldots) .
$$

From the boundary condition

$$
U_{n}(R, \varphi)=0 \quad 0<\varphi<2 \pi,
$$

it follows that

$$
A_{n k}=\frac{\alpha_{k} g}{2 n+4-\lambda_{k}^{2}} R^{2-p_{n k}} \quad(k=1,2, \ldots) .
$$

Combination of Eqs. (14) and (18) with Eq. (10) leads to the results

$$
U_{n}(r, \varphi)=\sum_{k=1}^{\infty} \frac{\alpha_{k} g}{2 n+4-\lambda_{k}^{2}}\left[R^{2}\left(\frac{r}{R}\right)^{p_{n k}}-\left(\frac{r}{R}\right)^{n} r^{2}\right] \sin \lambda_{k} \varphi .
$$

The determination of the torsion function $\omega_{n}=\omega_{n}(r, \varphi)$ is based on the solution of system of differential equations (9). Assuming that $\omega_{n}(0,0)=0$ and starting from the following equation (see Fig. 2)

$$
\omega_{n}(r, \varphi)=\int_{\frac{\partial Q}{O Q}}\left(\frac{\partial \omega}{\partial r} \mathrm{~d} r+\frac{\partial \omega_{n}}{\partial \varphi} \mathrm{d} \varphi\right)+\int_{\widehat{Q O}}\left(\frac{\partial \omega}{\partial r} \mathrm{~d} r+\frac{\partial \omega_{n}}{\partial \varphi} \mathrm{d} \varphi\right),
$$

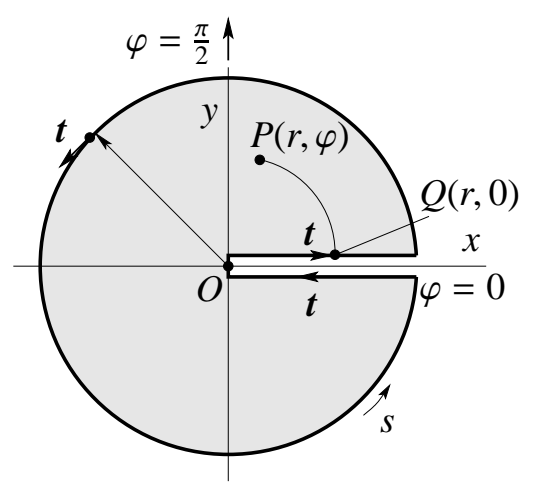

Fig. 2. Determination of torsion function. 
88 Saint-Venant Torsion of a Functionally Graded Circular Bar with a Radial Slit

where the line integrals in (20) are taken over the line segment $\overline{O Q}$ and circular segment $\widehat{Q P}$ (Fig. 2) and inserting the expressions of partial derivatives of $\omega_{n}=$ $\omega_{n}(r, \varphi)$ in terms of $U_{n}=U_{n}(r, \varphi)$ which are given by Eq. (9) we get

$$
\begin{aligned}
\omega_{n 1}(r, \varphi)=\sum_{k=1}^{\infty}\left[\frac{A_{n k}}{g} \frac{r^{p_{n k}}}{p_{n k}-n}\left(\frac{R}{r}\right)^{n}-\frac{\lambda_{k} \alpha_{k} r^{2}}{4 n+8-2 \lambda_{k}^{2}}-\right. \\
\left.\frac{A_{n k}}{g} \frac{p_{n k}}{\lambda_{k}} r^{p_{n k}}\left(\frac{R}{r}\right)^{n}+\frac{\alpha_{k}(n+2) r^{2}}{\left(2 n+4-\lambda_{k}^{2}\right) \lambda_{k}}\right]
\end{aligned}
$$

$$
\omega_{n 2}(r, \varphi)=\sum_{k=1}^{\infty}\left[\frac{A_{n k} p_{n k}}{g \lambda_{k}}\left(\frac{R}{r}\right)^{n} r^{p_{n k}}-\frac{\alpha_{k}(n+2) r^{2}}{\left(2 n+4-\lambda_{k}^{2}\right)}\right] \cos \lambda_{k} \varphi-r^{2} \varphi
$$

\section{Formulae of Shearing Stresses and Torsion Rigidity}

Application of formulae (4) gives the next results for the shearing stresses

$$
\begin{gathered}
\tau_{n r z}(r, \varphi)=\vartheta \sum_{k=1}^{\infty} \frac{\alpha_{k} \lambda_{k} g}{2 n+4-\lambda_{k}^{2}}\left[\frac{R^{2}}{r}\left(\frac{r}{R}\right)^{p_{n k}}-\left(\frac{r}{R}\right)^{n} r\right] \cos \lambda_{k} \varphi \\
\tau_{n \varphi z}(r, \varphi)=-\vartheta \sum_{k=1}^{\infty} \frac{\alpha_{k} g}{2 n+4-\lambda_{k}^{2}}\left[R p_{n k}\left(\frac{r}{R}\right)^{p_{n k}-1}\right. \\
\left.-(n+2)\left(\frac{r}{R}\right)^{n} r\right] \sin \lambda_{k} \varphi
\end{gathered}
$$

Substitution the expression of Prandtl's stress function into formula (6) yields the next result for the torsional rigidity $S_{n}$

$$
S_{n}=32 g R^{4} \sum_{k=1}^{\infty} \frac{\alpha_{k}\left(p_{k n}-n-2\right) \cos ^{2} \pi k}{(2 k-1)\left(p_{k n}+2\right)\left(4 k^{2}-4 k-8 n-15\right)(n+4)} .
$$

Equation (26) shows that the presented results are valid for $n \neq-4$. Let $s_{n}$ be defined as

$$
s_{n}=\frac{S_{n}}{32 g R^{4}} .
$$




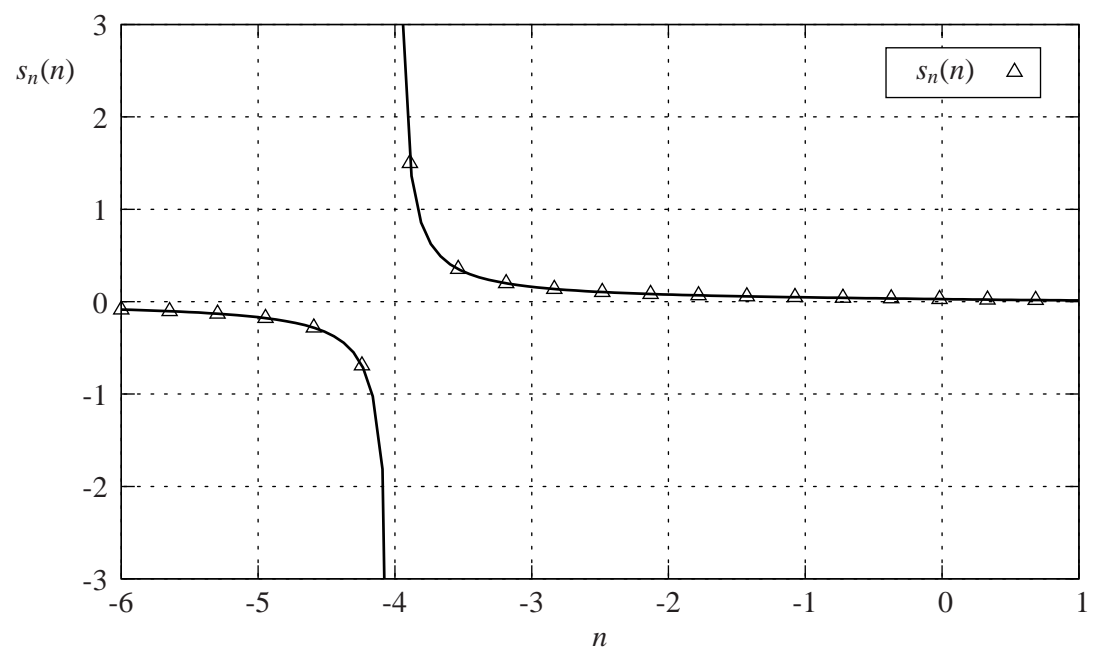

Fig. 3. The graph of $s_{n}$ as a function of $n$ for $-6<n<1$.

The graph of $s_{n}$ as a function of $n$ (Fig. 3) is supported that $S_{n}$ cannot be evaluated for $n=-4$ by the formula (26).

In the next torsional rigidity will be given in terms of torsion function. The shear stress vector $\boldsymbol{\tau}_{z}$ is defined as

$$
\boldsymbol{\tau}_{z}=\tau_{r z} \boldsymbol{e}_{r}+\tau_{\varphi z} \boldsymbol{e}_{\varphi}=\vartheta G(r)\left(\nabla \omega+\boldsymbol{e}_{z} \times \boldsymbol{r}\right) \quad \boldsymbol{r}=r \boldsymbol{e}_{r},
$$

where $\nabla$ is the Hamilton differential operator (del operator)

$$
\nabla=\frac{\partial}{\partial r} \boldsymbol{e}_{r}+\frac{1}{r} \frac{\partial}{\partial \varphi} \boldsymbol{e}_{\varphi}
$$

In Eq. (28) the cross between two vectors denotes their vectorial product.

The equilibrium equation and stress boundary condition for Saint-Venant torsion can be formulated as

$$
\nabla \cdot \boldsymbol{\tau}_{z}=0 \quad(r, z) \in A \quad \boldsymbol{n} \cdot \boldsymbol{\tau}_{z}=0 \quad(r, z) \in \partial A,
$$

where the dot denotes the scalar product of two vectors and $\boldsymbol{n}$ is the unit normal vector to the boundary curve $\partial A$ (Fig. 1). Eq. (30) in detailed form is as follows

$$
\begin{gathered}
\nabla \cdot\left(G(r)\left[\nabla \omega+\boldsymbol{e}_{z} \times \boldsymbol{r}\right]\right)=0 \quad(r, z) \in A, \\
\boldsymbol{n} \cdot\left(\nabla \omega+\boldsymbol{e}_{z} \times \boldsymbol{r}\right)=0 \quad(r, z) \in \partial A .
\end{gathered}
$$


90 Saint-Venant Torsion of a Functionally Graded Circular Bar with a Radial Slit

The torsional rigidity in terms of $\omega=\omega(r, \varphi)$ can be computed from the next formula

$$
\begin{array}{r}
S=\frac{T}{\vartheta}=\boldsymbol{e}_{z} \cdot \int_{A} G(r) \boldsymbol{R} \times\left(\nabla \omega+\boldsymbol{e}_{z} \times \boldsymbol{r}\right) \mathrm{d} A=\int_{A} G(r)\left(\boldsymbol{e}_{z} \times \boldsymbol{r}\right) \cdot\left(\nabla \omega+\boldsymbol{e}_{z} \times \boldsymbol{r}\right) \mathrm{d} A \\
=\int_{A} G(r)\left(\boldsymbol{e}_{z} \times \boldsymbol{r}\right) \cdot \nabla \omega \mathrm{d} A+\int_{A} G(r)\left(\boldsymbol{e}_{z} \times \boldsymbol{r}\right) \cdot\left(\boldsymbol{e}_{z} \times \boldsymbol{r}\right) \mathrm{d} A \\
=\int_{A} G(r)\left(\boldsymbol{e}_{z} \times \boldsymbol{r}\right) \cdot \nabla \omega \mathrm{d} A+\int_{A} G(r) r^{2} \mathrm{~d} A .
\end{array}
$$

From Eqs. (31) and (32) it follows that

$$
\begin{aligned}
\int_{A} \omega \nabla \cdot[G(r)(\nabla \omega & \left.\left.+\boldsymbol{e}_{z} \times \boldsymbol{r}\right)\right] \mathrm{d} A=\int_{\partial A} \omega \boldsymbol{n} \cdot G(r)\left(\nabla \omega+\boldsymbol{e}_{z} \times \boldsymbol{r}\right) \mathrm{d} s \\
& -\int_{A} G(r)|\nabla \omega|^{2} \mathrm{~d} A-\int_{A} G(r) \nabla \omega \cdot\left(\boldsymbol{e}_{z} \times \boldsymbol{r}\right) \mathrm{d} A=0,
\end{aligned}
$$

that is

$$
\int_{A} G(r) \nabla \omega \cdot \boldsymbol{e}_{z} \times \boldsymbol{r} \mathrm{d} A=-\int_{A} G(r)|\nabla \omega|^{2} \mathrm{~d} A .
$$

Substituting Eq. (35) into Eq. (33) we got

$$
S=\int_{A} G r^{2} \mathrm{~d} A-\int_{A} G(r)|\nabla \omega|^{2} \mathrm{~d} A .
$$

Simple computation gives the next results

$$
\begin{aligned}
\int_{A} \nabla \omega \cdot\left(G(r) \boldsymbol{e}_{z} \times \boldsymbol{r}\right) \mathrm{d} A & =\int_{A} \nabla \cdot\left(\omega G(r) \boldsymbol{e}_{z} \times \boldsymbol{r}\right) \mathrm{d} A-\int_{A} \omega \nabla \cdot\left(G(r) \boldsymbol{e}_{\varphi}\right) \mathrm{d} A \\
& =-\int_{\partial A} \omega\left(G(r)\left(\boldsymbol{n} \times \boldsymbol{e}_{z}\right)\right) \cdot \boldsymbol{r} \mathrm{d} s=-\int_{\partial A} \omega G(r) \boldsymbol{t} \cdot \boldsymbol{r} \mathrm{d} s .
\end{aligned}
$$

In Eq. (37) $t$ is the tangential unit vector to the boundary curve $\partial A, s$ is an arc-length defined on $\partial A$ (Fig. 2).

Previously, we use the equation

$$
\nabla \cdot\left(G(r) \boldsymbol{e}_{\varphi}\right)=\frac{\partial G}{\partial r} \boldsymbol{e}_{r} \cdot \boldsymbol{e}_{\varphi}-\frac{G(r)}{r} \boldsymbol{e}_{\varphi} \cdot \boldsymbol{e}_{r}=0 .
$$


The combination of Eq. (33) with Eq. (37) gives

$$
S=\int_{A} G(r) r^{2} \mathrm{~d} A-\int_{\partial A} \omega r G(r) \boldsymbol{t} \cdot \boldsymbol{e}_{r} \mathrm{~d} s .
$$

Here, we note formula (39) is a new formula of the torsional rigidity for radially nonhomogeneous cylindrical bar with radial slit. Application of formula (39) to the case of power law radial inhomogeneity leads to the next result

$$
S_{n}=\int_{A} g\left(\frac{r}{R}\right)^{n} \mathrm{~d} A-\int_{0}^{R} g\left(\frac{r}{R}\right)^{n} r(\omega(r, 0)-\omega(r, 2 \pi)) \mathrm{d} r .
$$

From the formulae of shearing stresses it follows that the shearing stresses have singularity at the tip of slit $(r=0)$ for $-4<n<0.75$ A detailed computation gives the next results

$$
\tau_{r z n}(r, \varphi)=\frac{16 \vartheta g R}{(8 n+15) \pi}\left[\left(\frac{r}{R}\right)^{0.5\left(n+\sqrt{n^{2}-1}-2\right)}\right] \cos \frac{\varphi}{2}+\text { regular terms },
$$

$$
\tau_{\varphi z n}(r, \varphi)=-\frac{-8 \vartheta g R}{\left(2 n+\frac{15}{4}\right) \pi}\left[\left(\frac{r}{R}\right)^{0.5\left(n+\sqrt{n^{2}+1}-2\right)}\right] \sin \frac{\varphi}{2}+\text { regular terms }
$$

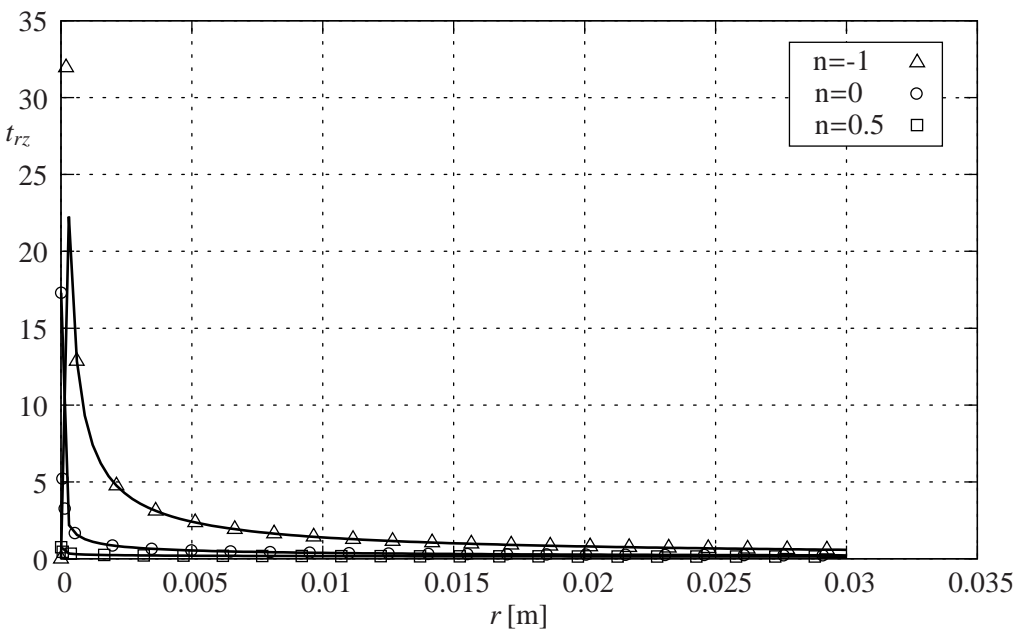

Fig. 4. Singularity of radial shearing stresses. 


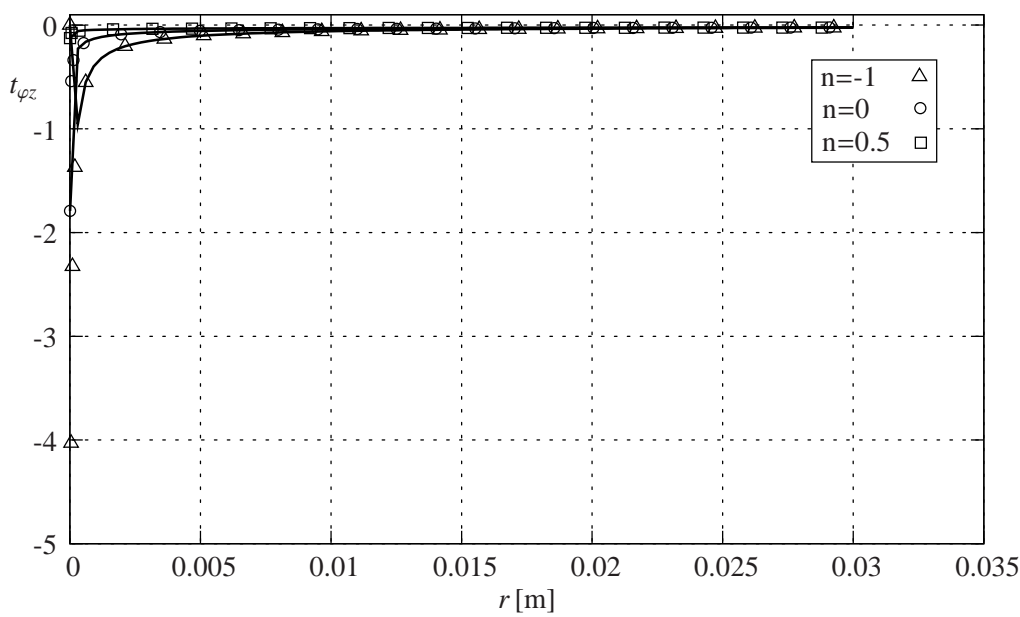

Fig. 5. Singularity of circumferential shear stresses.

Let

$$
t_{r z n}(r)=\frac{\left(\frac{r}{R}\right)^{0.5\left(n+\sqrt{n^{2}+1}-2\right)}}{(4 n+7.5) \pi} \quad t_{\varphi z n}(r)=\frac{\left(\frac{r}{R}\right)^{0.5\left(n+\sqrt{n^{2}+1}-2\right)}}{(2 n+7.5) \pi} .
$$

The singularity of stress field is illustrated in Fig. 4 and Fig. 5 some different value of power index.

\section{EXAMPLES}

The following numerical data are used in the example

$$
R=0.06 \mathrm{~m} \quad g=0.8 \times 10^{10} \mathrm{~Pa} \quad \vartheta=10^{-2} 1 / \mathrm{m} \quad n=2 .
$$

The plots of stress function as a function of $r$ at $\varphi=\frac{\pi}{2}$ are presented in Fig. 6 for the different values of power index $n$. The graphs of stress function as a function of $\varphi$ at $r=\frac{R}{2}$ are shown in Fig. 7 for different values of power index $n$. The torsional rigidity as a function of power index $n$ is given by in Fig. 8. The torsion function as a function of $r$ at $\varphi=\frac{\pi}{2}$ for different values of power index $n$ is shown in Fig. 9. Fig. 10 illustrates the graphs of torsion function as a function of $\varphi$ at $r=\frac{R}{2}$ for $\alpha=-1,0,1,2$. The contour lines of stress function for $n=2$ is presented in Fig. 11. Fig. 12 shows the contour lines of torsion function for $n=2$. Fig. 17 shows the surface of torsion function for $n=2$ as a function of $r$ and $\varphi$ in the threedimensional space. Fig. 18 illustrates the surface of Prandtl's stress function as a function of $r$ and $\varphi$ in the three-dimensional space for $n=2$. 


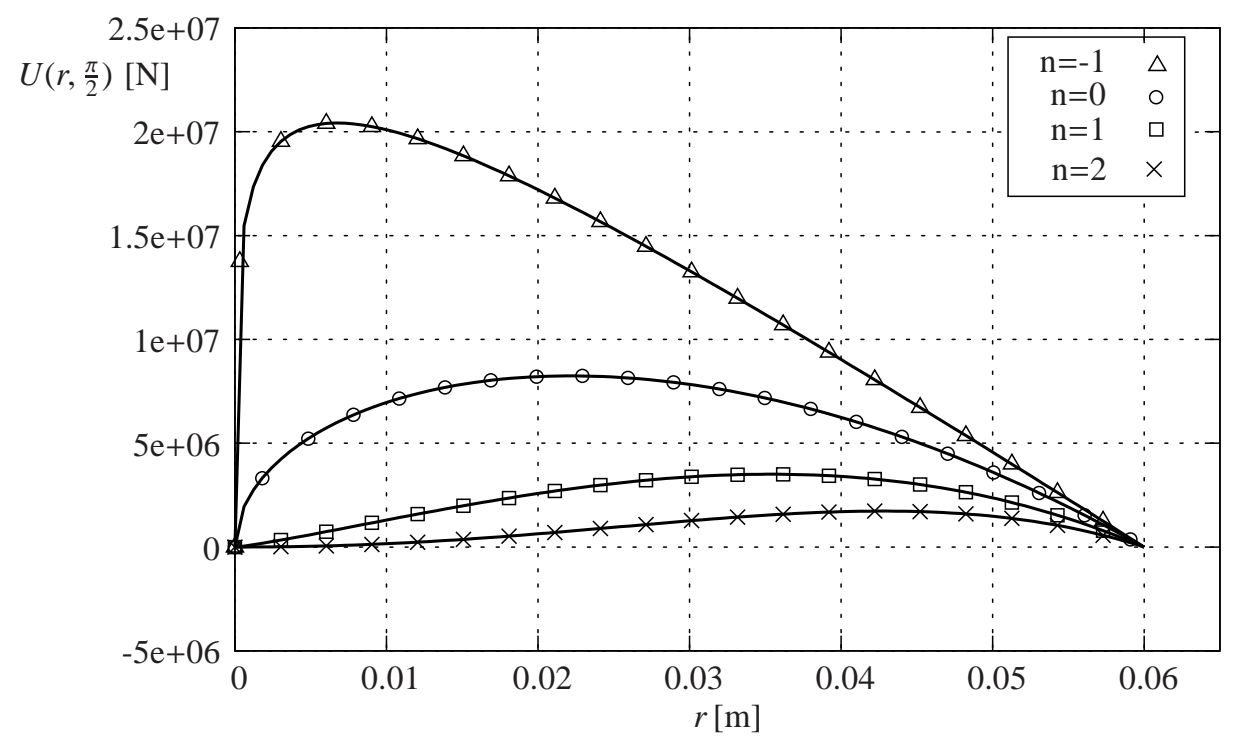

Fig. 6. Plots of stress function as a function of $r$ at $\varphi=\pi / 2$.

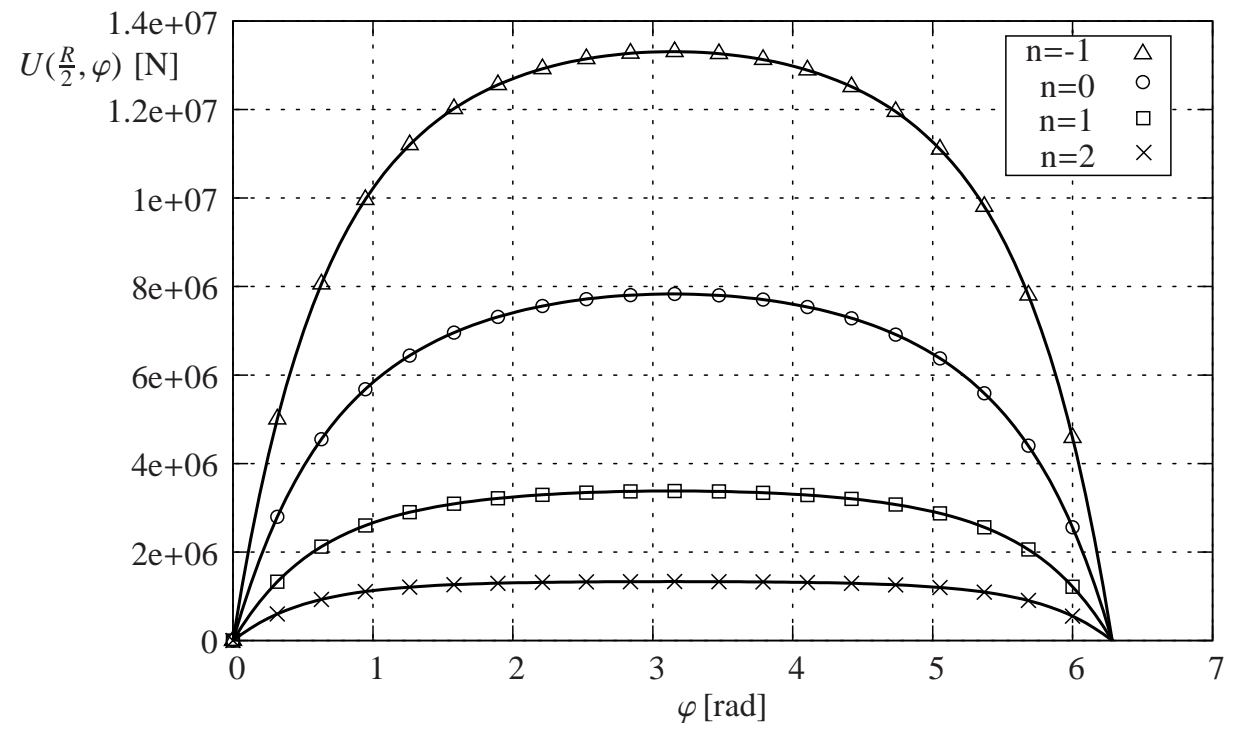

Fig. 7. Plots of stress function as a function of $\varphi$ at $r=R / 2$. 
94 Saint-Venant Torsion of a Functionally Graded Circular Bar with a Radial Slit

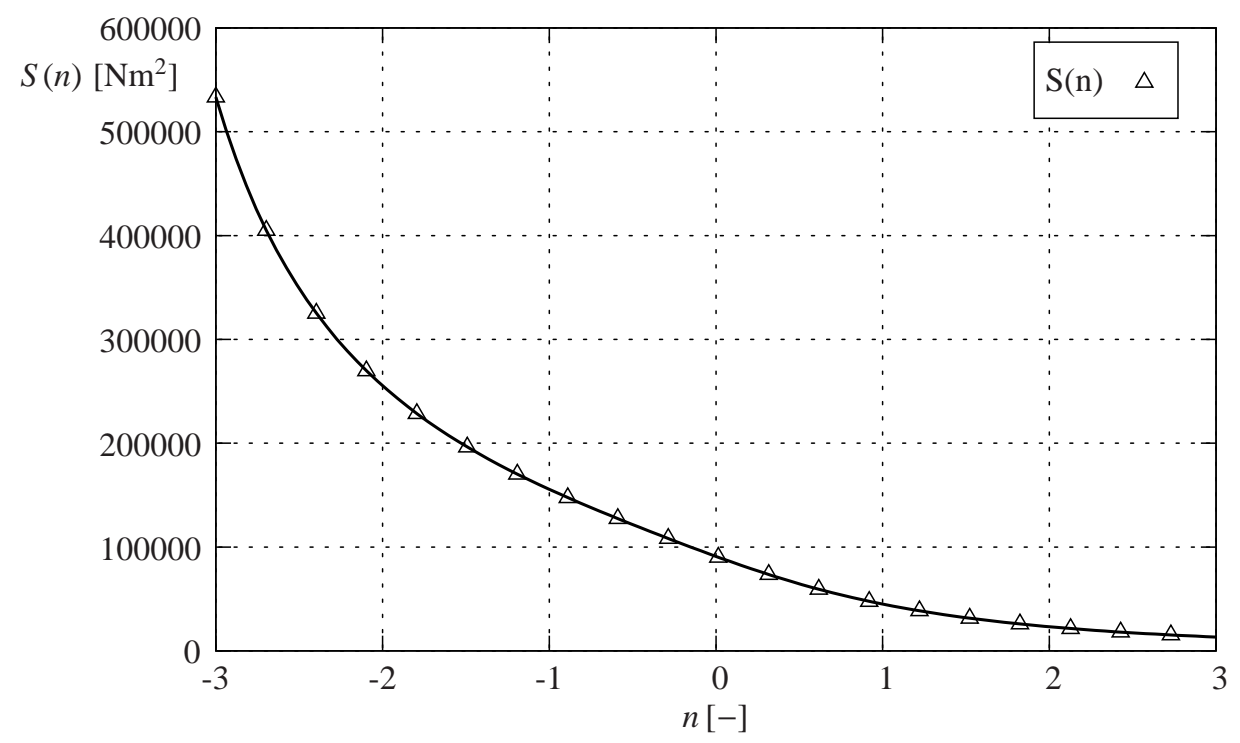

Fig. 8. Plots of the torsional rigidity as a function of $n$ for $n=[-3,3]$.

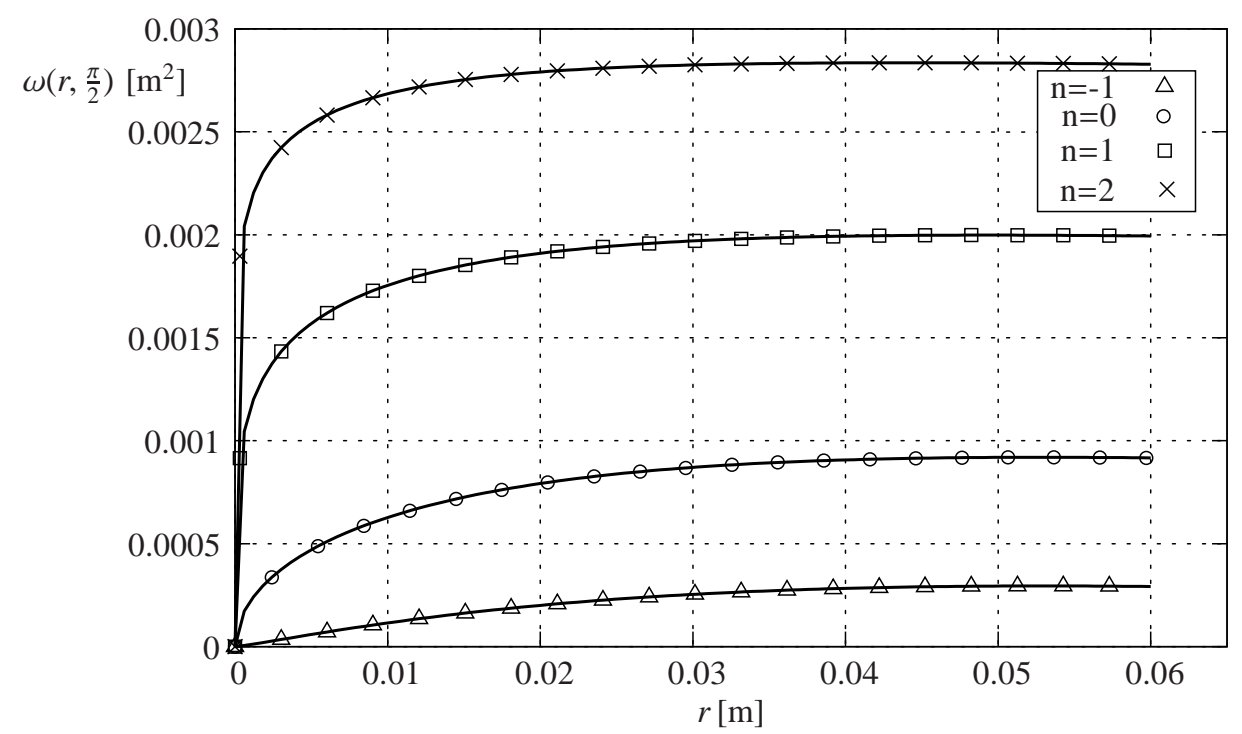

Fig. 9. Plots of torsion function as a function of $r$ at $\varphi=\pi / 2$. 


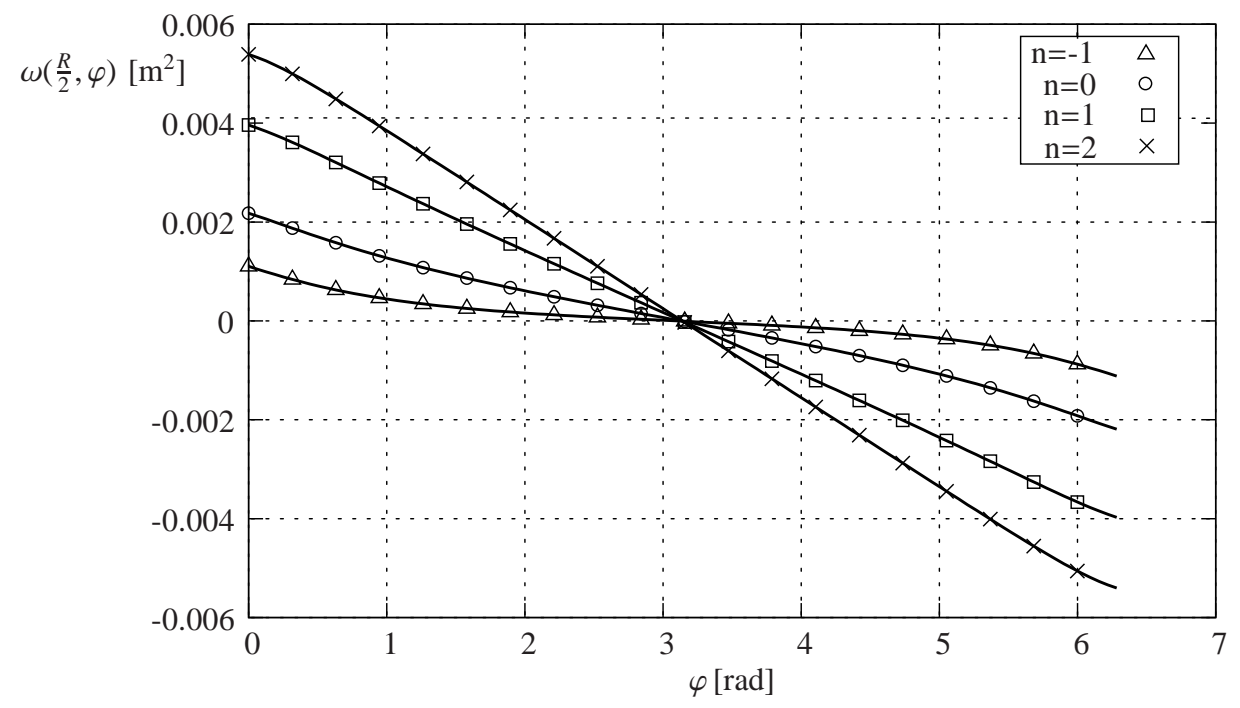

Fig. 10. Plots of torsion function as a function $\varphi$ at $r=R / 2$.

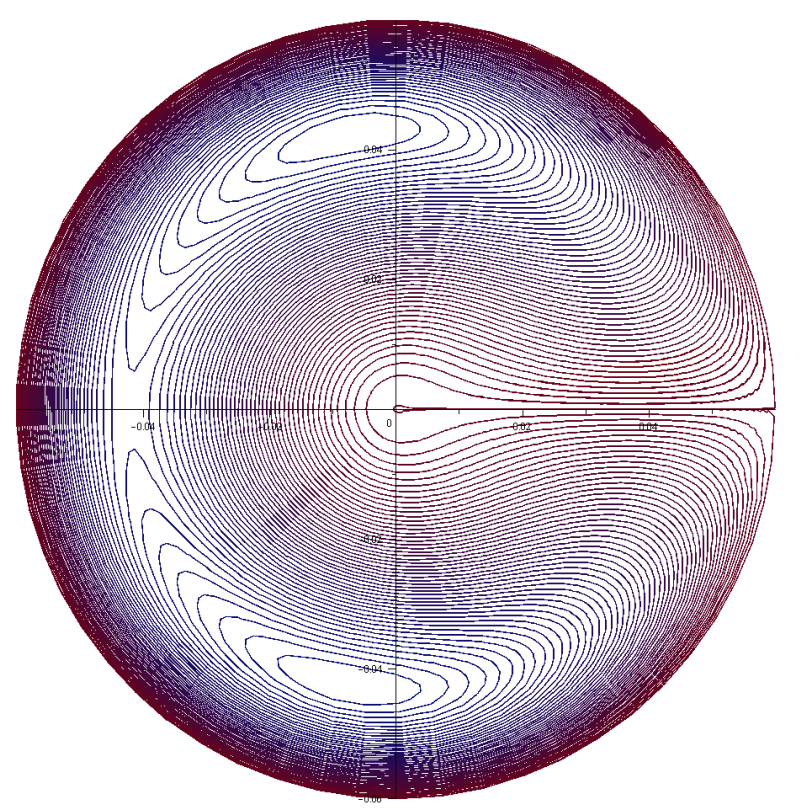

Fig. 11. The contour lines of Prandtl's stress function for $n=2$. 


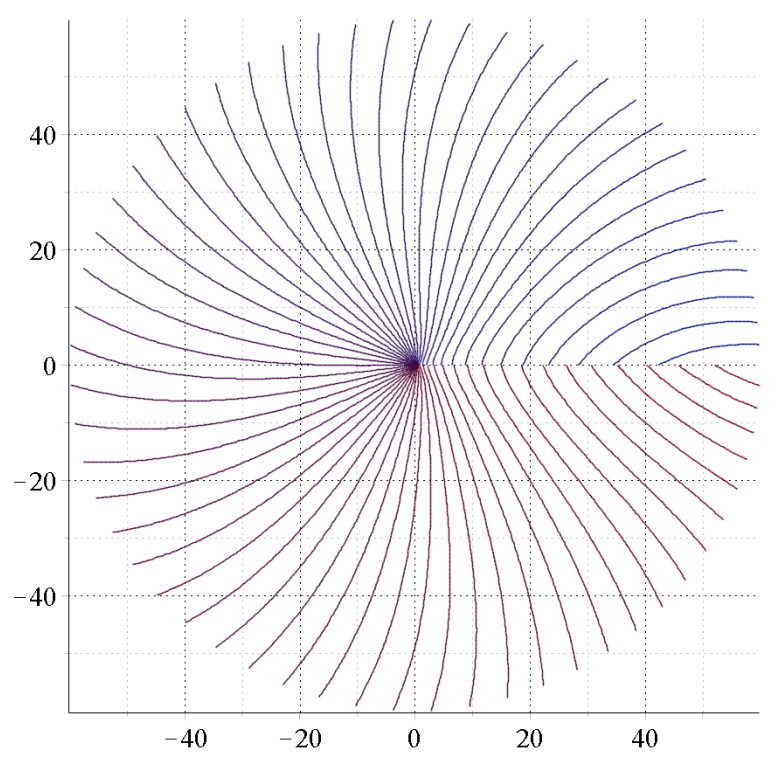

Fig. 12. The contour lines of the torsion function for $n=2$.

The shearing stresses $\tau_{x z}\left(r, \frac{\pi}{2}\right)$ and $\tau_{x z}(r, 2 \pi)$ as a function of $r$ in comparison with FEM (Abaqus) solution are shown in Fig. 13.

The graphs of shear stresses $\tau_{y z}$ for $\varphi=\frac{\pi}{2}$ and $\varphi=2 \pi$ are presented in Fig. 14 in comparison with FEM (Abaqus) solution. Fig. 15 shows the graphs of torsion function for $\varphi=\frac{\pi}{6}$ and $\varphi=\frac{\pi}{2}$ obtained from formula (22) of the present paper and FEM solution.

Illustration of torsion function on axis $\varphi=0(0 \leq x \leq R)$ and on axis $\varphi=2 \pi$ $(0 \leq x \leq R)$ comparison with FEM (Abaqus) solution are presented in Fig. 16.

Application of formula (26) gives the next result for torsional rigidity

$$
S=23237.56 \mathrm{Nm}^{2}
$$

and from the formula (40) it follows that

$$
S=23237.56 \mathrm{Nm}^{2} .
$$

By the aid of formula (40) we can get the torsional rigidity from the numerical results of FEM computations, in the present case

$$
S=23506.05 \mathrm{Nm}^{2} .
$$




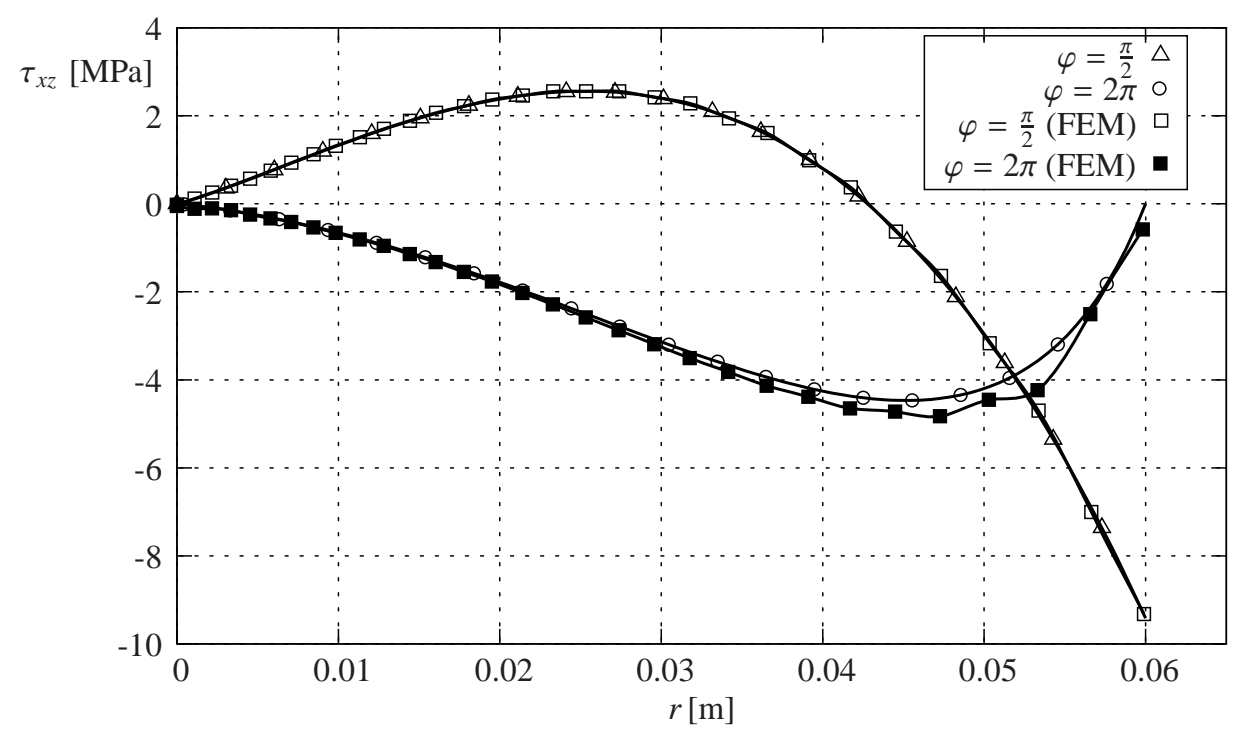

Fig. 13. Plots of shear stresses $\tau_{x z}$.

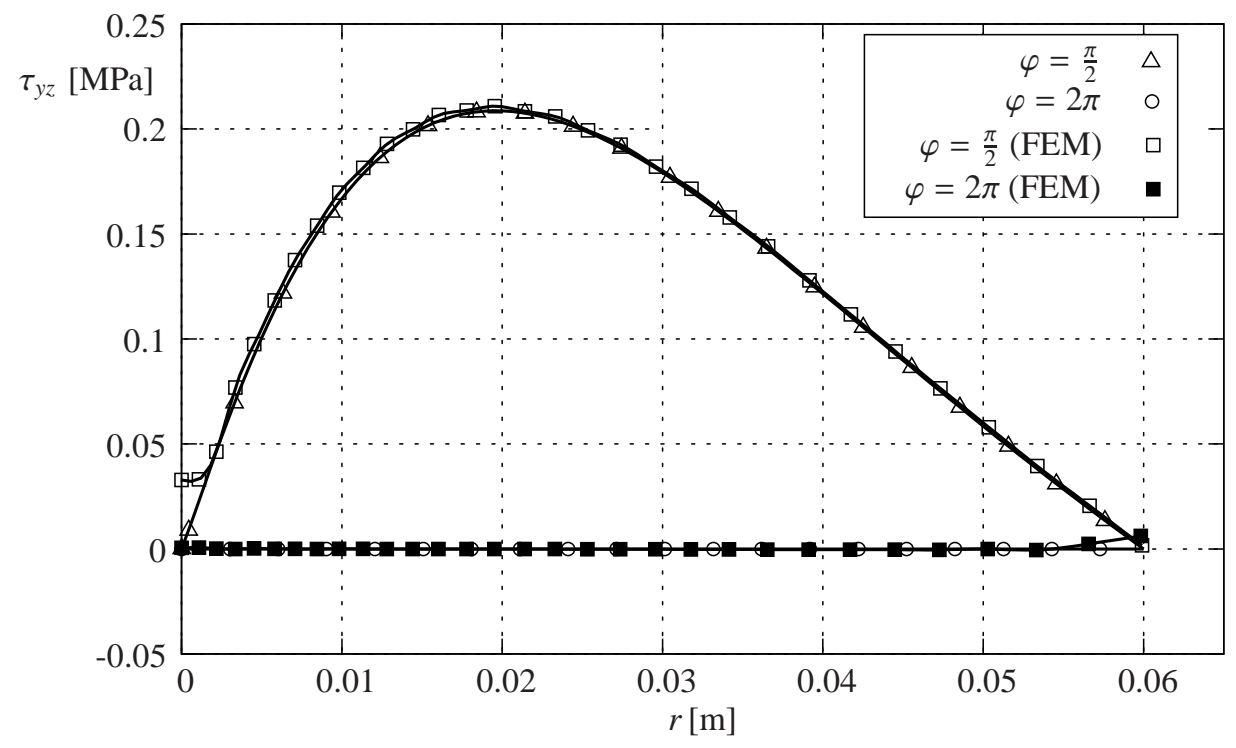

Fig. 14. Plots of shear stresses $\tau_{y z}$. 
98 Saint-Venant Torsion of a Functionally Graded Circular Bar with a Radial Slit

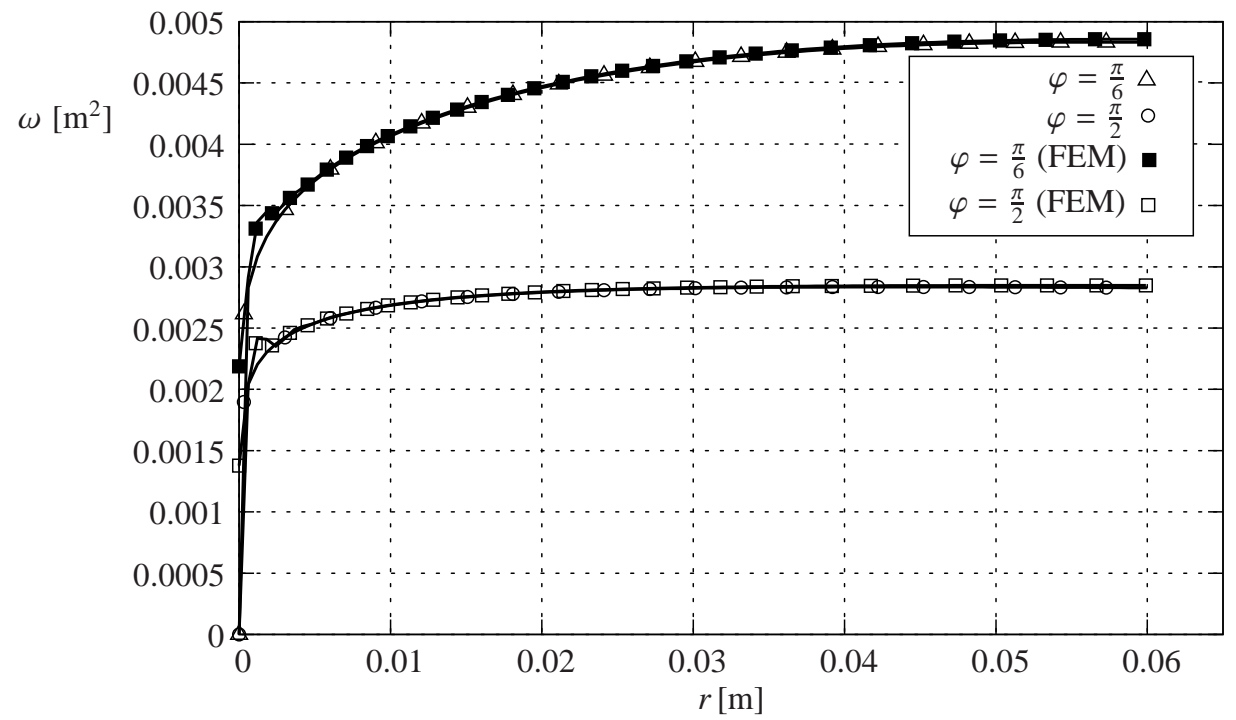

Fig. 15. Plots of torsion functions.

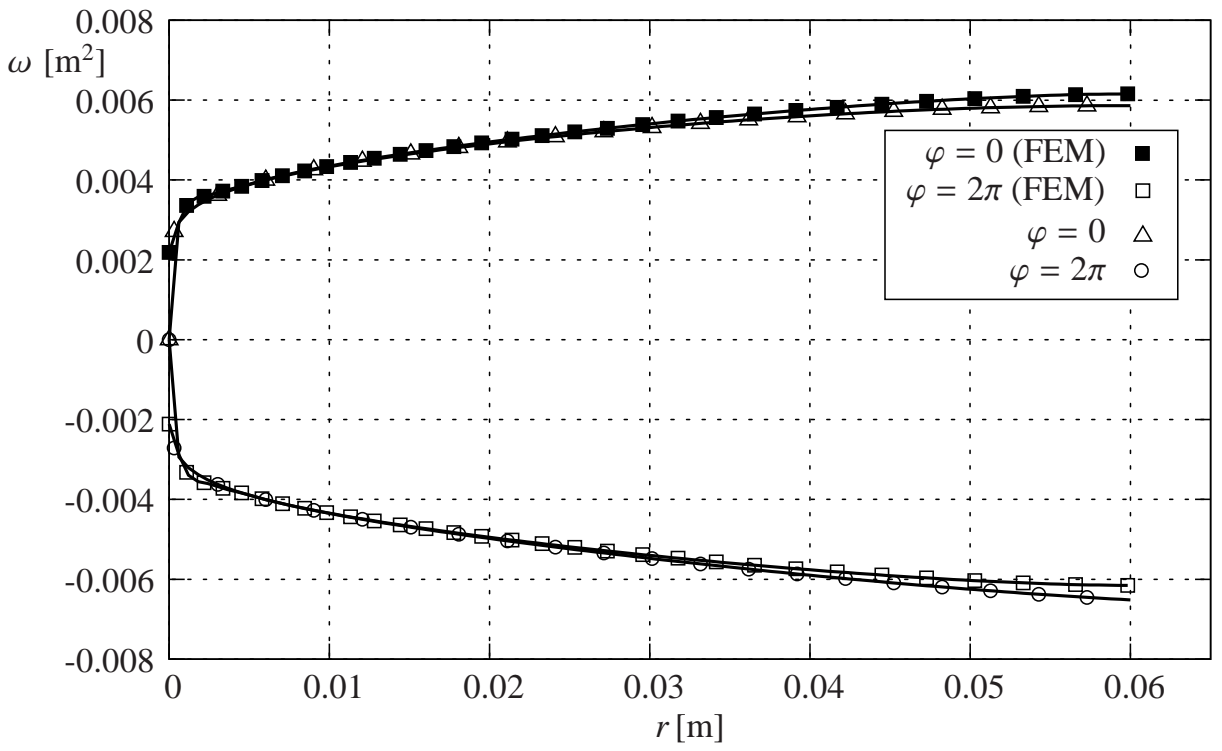

Fig. 16. Plots of torsion functions. 


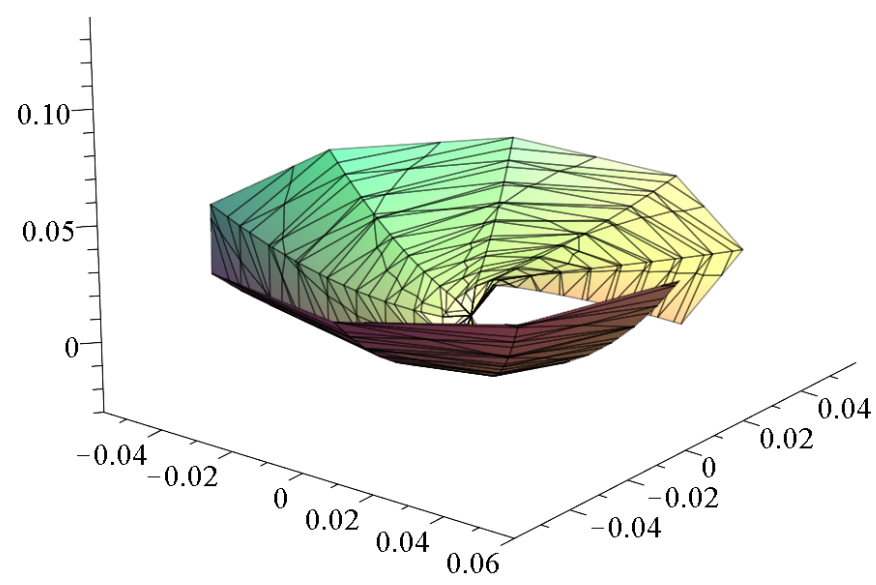

Fig. 17. The graph of torsion function for $n=2$.

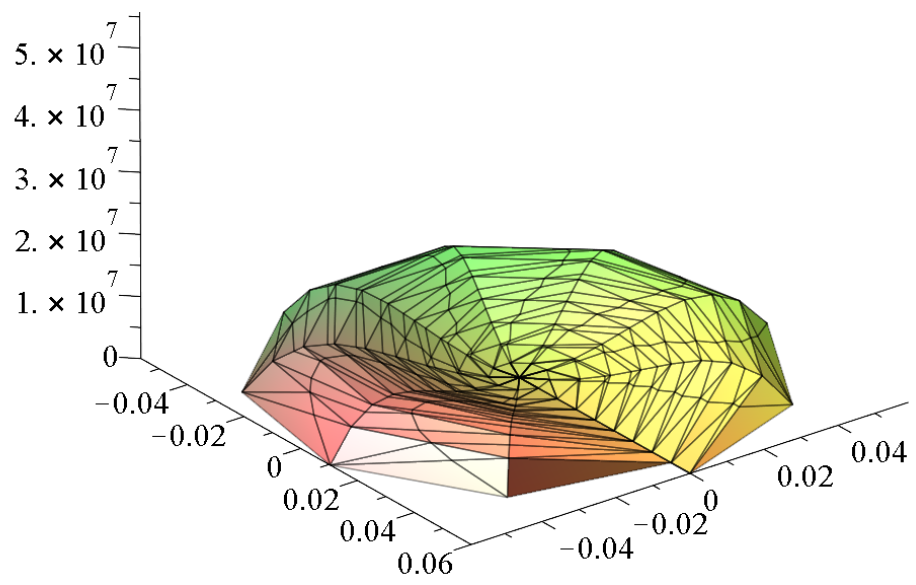

Fig. 18. The graph of Prandtl's stress function for $n=2$.

\section{CONCLUSIONS}

An analytical solution of Saint-Venant torsion for nonhomogeneous circular with a slit extending in radial direction to the axis of bar is presented. The nonhomogeneity of the isotropic, linearly elastic bar is described by a power law function of radial coordinate. Solutions in chosen form of the radial nonhomogeneity are given for Prandtl's stress function, torsion function, shearing stresses and torsional rigidity. Paper also deals with the analysis of dependence of Prandtl's stress function, torsion 
function and torsional rigidity as function of power law index of shear modulus. Results are obtained by the presented analytical method are in good agreement with the FEM (Abaqus) solutions.

\section{ACKNOWLEDGEMENTS}

The described article/presentation/study was carried out as part of the EFOP-3.6.116-00011 Younger and Renewing University Innovative Knowledge City institutional development of the University of Miskolc aiming at intelligent specialisation project implemented in the framework of the Szechenyi 2020 program. The realization of this project is supported by the European Union, co-financed by the European Social Fund. This research was (partial) carried out in the framework of Center of Excellence of Innovative Engineering Design and Technologies at the University of Miskolc and supported by the National Research Development and Innovation Office - NKFIH K115701.

\section{REFERENCES}

[1] W.O. NowACKI (1953) Pewnych Przypadkach Skreceina Prestow. Archiwum Mechaniki Stosowang 5 21-46.

[2] Nowacki, W. O. Certain Classes of Torsion of Bars. Bulletin de l'Académie Polonaise des Sciences, 1 (1953), 18-23.

[3] Nowacki, W. O. Theory of Elasticity, Fizmathghiz, Moscow, 1983, (in Russian).

[4] Arutyunyan, N. H., Abramyan, B. L. Torsion of Elastic Bodies. Fizmathghiz, Moscow, 1963, (in Russian).

[5] Albas, J. B. On the Torsion of Cylindrical Bar. Proc. Konink. Nederl Acad. van Wetenschappen. 64, (1961), 47-51.

[6] Saint-Venant, B. Sur le Torsion des Prismes á Base Mixtiligue et sur une Singularité que Peuwent Offir Certaines Empois de la Coordinnée Logarithmique du Syséme Clindricque Isotherme de Lamé. Comptes Rendus des Scéances de l'Académie des Sciences. 87, (1878), 849-854.

[7] Saint-Venant, B. Examples du Calcul de la Torsion des Prismes á Base Mixtiligne. Comptes Rendus des Scéances de l' Académie des Sciences 87, (1878), 893-899.

[8] Suresh, S.M. Fundamentals of Functionally Graded Materials. IOM Communications Limited, London, 1998.

[9] Shen, H.S. Functionally Graded Materials. Nonlinear Analysis of Plates and Shells. CRC Press, New-York, 2009.

[10] Leknitskii, S.G. Theory of Elasticity of an Anisotropic Body. Mir. Publishers, Moscow, 1981 (In Russian).

[11] Rand, O., Rovenski, V. Analytical Methods in Anisotropic Elasticity with Symbolic Tools. Birkhammer, Boston, 2005. 
[12] Horgan, C.O. and Chan, A.M. Torsion of functionally graded isotropic linearly elastic bars. Journal of Elasticity. 52(2), (1998) 181-199

[13] Arghavan, S. and Hematiyan, M.R. Torsion of functionally graded hollow tubes. European Journal of Mechanics-A/Solids. 28(3), (2009), 551-559.

[14] Ecsedi, I. Some analytical solutions for Saint-Venant torsion of non-homogeneous cylindrical bars, European Journal of Mechanics-A/Solids. 28(5), (2009), 985-990

[15] Hematiyan, M.R. and Estakhrian, E., Torsion of functionally graded open-section members, International Journal of Applied Mechanics. 4(2), (2012), p.1250020 